\title{
Postsynthetic Metalated MOFs as Atomically Dispersed Catalysts for Hydroformylation Reactions
}

\author{
Panjuan Tang, Stefano Paganelli, Francesco Carraro,* Matias Blanco, Raffaele Riccò, Carla Marega, \\ Denis Badocco, Paolo Pastore, Christian J. Doonan, and Stefano Agnoli*
}

Cite This: ACS Appl. Mater. Interfaces 2020, 12, 54798-54805

Read Online

\section{ACCESS | Lill Metrics \& More | 回 Article Recommendations ｜（） Supporting Information}

ABSTRACT: A manganese-based metal-organic framework with dipyrazole ligands has been metalated with atomically dispersed $\mathrm{Rh}$ and Co species and used as a catalyst for the hydroformylation of styrene. The Rh-based materials exhibited excellent conversion at $80{ }^{\circ} \mathrm{C}$ with complete chemoselectivity, high selectivity for the branched aldehyde, high recyclability, and negligible metal leaching.

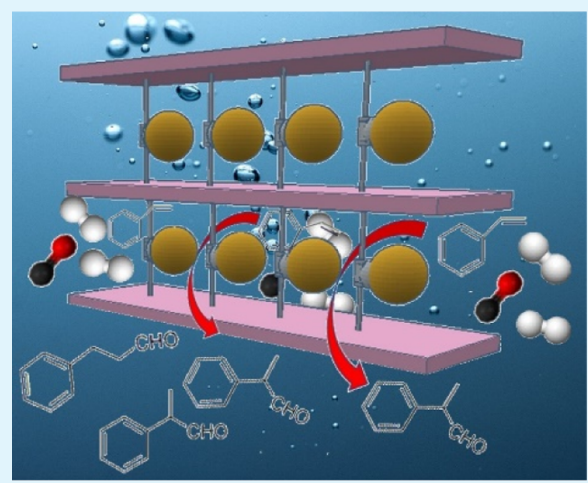

KEYWORDS: metal-organic frameworks, hydroformylation of olefins, heterogeneous catalysis, single-atom catalysts, rhodium coordination compounds

\section{INTRODUCTION}

Hydroformylation of alkenes for the production of aldehydes through the so-called oxo-process, (ca. 10 million ton/year) is one of the most important industrial reactions catalyzed by homogeneous metal complexes. ${ }^{1-3}$ Rhodium compounds, possessing phosphines or phosphite ligands, are the most efficient catalysts in terms of both activity and selectivity; furthermore, they operate under mild conditions. ${ }^{4}$ However, the oxo-process still suffers from some technical difficulties such as the separation of the expensive catalyst from the product mixture. With the aim of combining the high activity and selectivity of homogeneous catalysts and the easy separation and recovery associated with heterogeneous systems, researchers have sought to immobilize molecular hydroformylation catalysts on solid supports. Within this context, one of the most convenient and feasible approaches toward the development of improved catalysts is to incorporate metal complexes within the pores of inorganic materials to result in heterogenizing homogeneous catalysts. ${ }^{5-7}$ Porous solids have been widely explored as catalyst supports because of their large internal surface area and thermal stability. ${ }^{8-10}$ Among these, metal-organic frameworks (MOFs) are attracting significant interest because of their modular synthesis that allows for the precise control of pore size, chemical functionality, and topology. ${ }^{4,11}$ Indeed, many examples can be found, where the catalytic performance of MOFs can be enhanced by tuning the chemical environment within the pores. For example, incorporating nanoparticles and/or introducing homogeneous catalysts into a framework architecture has been shown to facilitate catalysis. ${ }^{4,12-15}$ To date, most attempts to combine Rh-based catalysts and MOFs have focused on dispersing $\mathrm{Rh}$ nanoparticles inside their pores. ${ }^{16-18}$ This approach, however, can result in a lack of structural homogeneity; moreover, a high-size dispersion of NPs and/or pore blockage can lead to the deactivation of a portion of the catalyst. ${ }^{19,20}$ An alternative strategy for supporting Rh-based catalysts in a microporous framework is the postsynthetic metalation of MOFs endowed with chelating ligands such as bipyridine ${ }^{21}$ or dipyrazole units. ${ }^{22}$ Under judicious conditions, this approach yields a highly homogeneous dispersion (atomically dispersed) of active sites within MOF pores. To this end, Bloch et al. synthesized a MOF, based on trinuclear $\mathrm{Mn}(\mathrm{II})$ nodes $\left(\mathrm{MnMOF}=\left[\mathrm{Mn}_{3}(\mathrm{~L})_{2}\left(\mathrm{~L}^{\prime}\right)\right]\right.$, where $\mathrm{LH}_{2}=$ bis(4-(4-carboxyphenyl)-1H-3,5dimethylpyrazolyl)methane); L' possesses a bis(3,5-dimethylpyrazol-1-yl)methane moiety that remains noncoordinated in the as-synthesized MOF and provides a flexible dipyrazole

Received: September 22, 2020

Accepted: November 13, 2020

Published: November 24, 2020 
chelating unit poised for postsynthetic metal binding. ${ }^{23}$ It was demonstrated that this MnMOF could be quantitatively metalated with different transition-metal cations (i.e., Co(II), $\mathrm{Cu}(\mathrm{II})$, and $\mathrm{Rh}(\mathrm{I})$ ) without the loss of the microporous structure of the material. The crystal structure of the postsynthetic metalated MnMOF was investigated via singlecrystal X-ray diffraction (SCXRD), and the presence of atomically dispersed transition-metal cations was demonstrated. ${ }^{23}$ Notably, the unique structure of this MOF affords only a single framework-bound coordination site per pore, thus minimizing the steric congestion within the "nanoreactor". Here, we synthesize MnMOF metalated with $\mathrm{Rh}(\mathrm{I})$ or $\mathrm{Co}(\mathrm{II})$ (starting from $\left[\mathrm{Rh}(\mathrm{CO})_{2} \mathrm{Cl}\right]_{2}$ or $\mathrm{CoCl}_{2} \cdot\left(\mathrm{H}_{2} \mathrm{O}\right)_{6}$ precursors, respectively) to obtain a framework replete with sites suitable for catalytic reactions (Figure 1). ${ }^{24}$ We tested the metalated

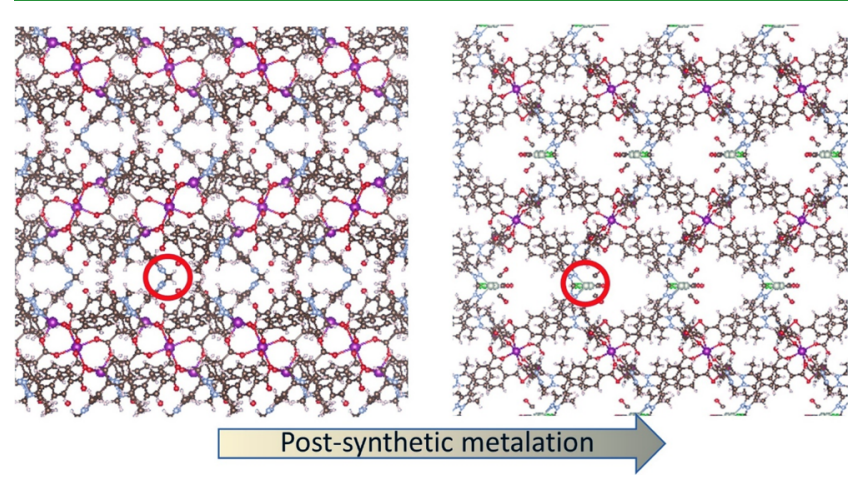

Figure 1. Portion of the structure of MnMOF (left) and of the postsynthetic metalated $\mathrm{Rh} / \mathrm{MnMOF}$ (right) viewed along the $c$ axis. The vacant (left) dipyrazole groups poised for postsynthetic metalation and their metalated counterpart (right) are highlighted with red circles. $(\mathrm{Mn}=$ purple; $\mathrm{O}=$ red; $\mathrm{C}=$ brown; $\mathrm{H}=$ white; $\mathrm{N}=$ light blue; $\mathrm{Rh}=$ gray; and $\mathrm{Cl}=$ green $)$.

MOFs (Rh/MnMOF, Co/MnMOF) for the hydroformylation of styrene, a widely studied model substrate for functionalized olefins. To the best of our knowledge, this reaction has not been explored for atomically dispersed catalysts that are an inherent part of the MOF structure. In addition, we synthesized the homogeneous counterpart of the investigated catalysts by means of metalation of the ester of the ligand molecule to assess the effect of the MOF pore environment on the catalytic activity.

\section{RESULTS AND DISCUSSION}

Following the previous report by Bloch et al., ${ }^{23}$ we synthesized the $\mathrm{Mn}(\mathrm{II})$-based MOF, $\left[\mathrm{Mn}_{3}(\mathrm{~L})_{2}\left(\mathrm{~L}^{\prime}\right)\right]$. In the $\mathrm{L}$ form, the carboxylate and pyrazole donors coordinate the $\mathrm{Mn}_{3}$ nodes. Conversely, in the $\mathrm{L}^{\prime}$ form, only the carboxylate donors are involved with the Mn coordination. Structurally, the MnMOF is composed of two-dimensional layers of trinuclear $\mathrm{Mn}_{3}(\mathrm{II})$ $(\mathrm{L})_{2}$ nodes pillared by the metal-free $\left(\mathrm{L}^{\prime}\right)$ form of the ligand (Figure 1). Therefore, for each metal node, $\mathrm{L}^{\prime}$ provides a flexible dipyrazole chelating unit poised for postsynthetic metal binding.

We synthesized two different metalated MnMOFs employing $\left[\mathrm{Rh}(\mathrm{CO})_{2} \mathrm{Cl}\right]_{2}(\mathrm{Rh} / \mathrm{MnMOF})$ and $\mathrm{CoCl}_{2} \cdot\left(\mathrm{H}_{2} \mathrm{O}\right)_{6}(\mathrm{Co} /$ MnMOF) as precursors. The XRD patterns of the metalated MOFs (Figures 2 and S1) are in accordance with the calculated XRD patterns reported in the literature for $\mathrm{Rh}(\mathrm{I})$ and $\mathrm{Co}$ (II) postsynthetic metalated MnMOF (Figures 2 and

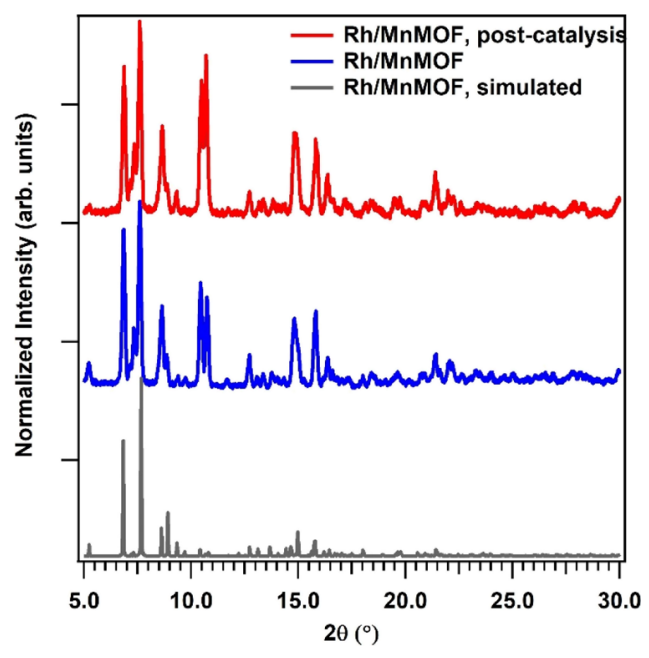

Figure 2. XRD patterns of the fresh and used $\mathrm{Rh} / \mathrm{MnMOF}$ and the simulated XRD pattern of Rh/MnMOF (from ref 23).

S1). ${ }^{23}$ Moreover, the XRD patterns exclude the presence of crystalline clusters of metallic or oxidized Rh or Co. According to the SCXRD data reported by Bloch et al., in the $\mathrm{Rh} /$ MnMOF, that is, MnMOF. $\left[\mathrm{Rh}(\mathrm{CO})_{2}\right]\left[\mathrm{RhCl}_{2}(\mathrm{CO})_{2}\right]$, one $\mathrm{Rh}(\mathrm{I})$ species presents a square planar geometry consisting of two nitrogen donors from the dipyrazole unit and two carbonyl ligands. In addition, a counterion (square planar $\left.\left[\mathrm{RhCl}_{2}(\mathrm{CO})_{2}\right]^{-}\right)$is present in the pores. In the case of $\mathrm{Co} /$ MnMOF, octahedral $\mathrm{Co}(\mathrm{II})$ species (MnMOF. $\left[\mathrm{Co}\left(\mathrm{H}_{2} \mathrm{O}\right)_{4}\right]$ $\mathrm{Cl}_{2}$ ) are present. $^{23}$ Energy-dispersive X-ray spectroscopy (EDX) analysis and inductively coupled plasma-mass spectrometry (ICP-MS) analysis (Table S1) confirmed that the metalation was quantitative and confirmed the composition expected for $\mathrm{Rh} / \mathrm{MnMOF}$ and $\mathrm{Co} / \mathrm{MnMOF}^{23}$ After metalation, the pillaring ligands undergo significant torsional rotation (as previously demonstrated via SCXRD. ${ }^{23}$ This causes a contraction of the two-dimensional planes, which is highlighted from the analysis of the XRD patterns and by the predominance of the (001) and (110) diffraction peaks in the metalated samples (at 6.8 and $7.7^{\circ}$, respectively) (Figures 2 and $\mathrm{S} 1$ ).

To assess the porosity of pristine and metalated MOFs, we measured $77 \mathrm{~K} \mathrm{~N}_{2}$ adsorption isotherms. The MnMOF isotherm profile shows a step between $P / P_{0}$ of $0.005-0.06$ that is attributed to the degree of structural flexibility (Figure 3 ) that is associated with the rotation of the pyrazole moieties and concomitant pore enlargement. ${ }^{23}$ A total $\mathrm{N}_{2}$ uptake of 250 $\mathrm{cm}^{3} \mathrm{~g}^{-1}$ (STP) was observed, and the BET analysis of data yielded a surface area of $723 \pm 5 \mathrm{~m}^{2} \mathrm{~g}^{-1}$. The postsynthetically metalated MOFs $\mathrm{Rh} / \mathrm{MnMOF}$ and Co/MnMOF both afforded type I isotherms with BET surface areas of $631 \pm 2$ and $839 \pm 1 \mathrm{~m}^{2} \mathrm{~g}^{-1}$, respectively (Figures 3, S2 and S3). These data confirm that the overall porosity is maintained subsequent to metalation. Furthermore, we note that the low-pressure step of the isotherm observed for the MnMOF disappears, as when the $\mathrm{Rh}(\mathrm{I})$ or $\mathrm{Co}(\mathrm{II})$ cations are coordinated by the dipyrazole chelating units, the structural flexibility of the framework is lost, an observation that is consistent with previous studies and with the changes observed in the diffraction patterns of the metalated samples. ${ }^{23}$

The structural integrity of metalated MOFs was further investigated by Raman spectroscopy (Figures 4 and S4). Compared with the pristine MnMOF, the Raman spectra of 


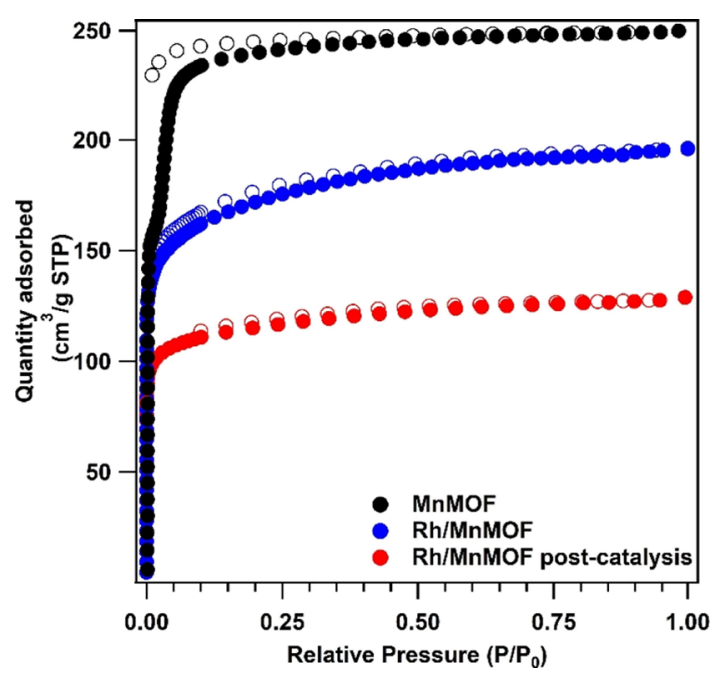

Figure 3. $77 \mathrm{~K} \mathrm{~N}_{2}$ adsorption isotherms of MnMOF and of fresh and used $\mathrm{Rh} / \mathrm{MnMOF}$.

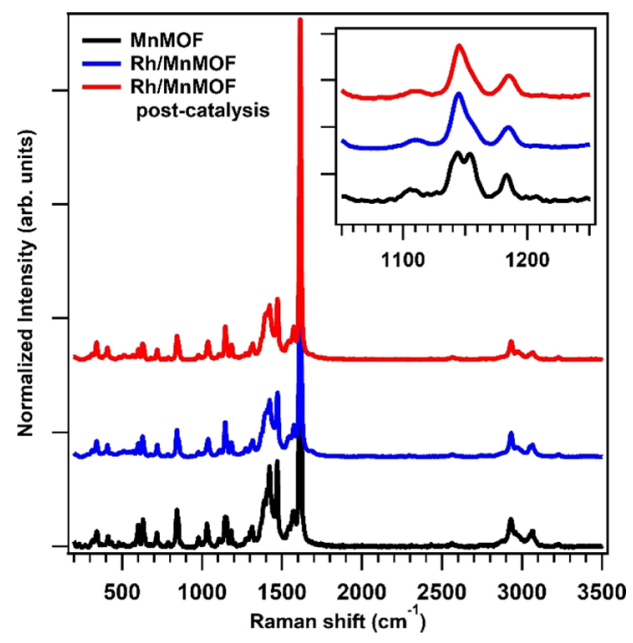

Figure 4. Raman spectra of MnMOF and of fresh and used $\mathrm{Rh} /$ MnMOF. The inset shows an enlarged vision of the Raman shift region pertaining to $\mathrm{N}-\mathrm{H}$ deformation.

$\mathrm{M} / \mathrm{MnMOFs}$ showed negligible changes in band intensity. The major differences in the regions $1610-1630 \mathrm{~cm}^{-1}(\nu(\mathrm{C}=\mathrm{N}))$, $1350-1450 \mathrm{~cm}^{-1}(\nu(\mathrm{N}=\mathrm{N})$ aromatic $), 1120-1170 \mathrm{~cm}^{-1}$ $(\mathrm{N}-\mathrm{H}$ deformation, inset Figure 4$)$, and $2900-3000 \mathrm{~cm}^{-1}$ $(\nu(\mathrm{C}-\mathrm{H}))$ can be attributed to metal coordination by the dipyrazole moieties. In addition, the Raman data also confirm the absence of $\mathrm{Mn}$ or $\mathrm{Rh} / \mathrm{Co}$ metal oxide clusters in the materials. ${ }^{25-27}$

The presence of Rh and Co only in the pores of the MOF as coordination compounds is also confirmed by photoemission measurements (Figures S5 and S6). The Rh 3d core level photoemission peak is centered at $308.2 \mathrm{eV}$, which is in agreement with other soft ligated $\mathrm{Rh}(\mathrm{I})$ species. ${ }^{28}$ The binding energy (BE) position of the Co $2 \mathrm{p}_{3 / 2}$ peak at $781 \mathrm{eV}$, as well as the position of its final state satellite, is indicative of $\mathrm{Co}$ (II) ions coordinated to very electronegative atoms. ${ }^{29}$ Moreover, the chemical composition deduced by the intensity of photoemission peaks (e.g., at a $\mathrm{Mn} / \mathrm{Rh} \mathrm{w} / \mathrm{w}$ ratio of $1.4 \pm$ $0.15)$ is in good agreement with the IC-MS and EDX data (Table S1), indicating the absence of any surface segregation of rhodium or cobalt on the surface. Therefore, we can conclude that the metalation procedure was successful and resulted in a homogeneous distribution of atomically dispersed catalytic sites within the porous structure of the MOF.

Next, we assessed the potential of $\mathrm{Rh} / \mathrm{MnMOF}$ and $\mathrm{Co} /$ MnMOF to catalyze the hydroformylation of styrene (Scheme 1), a widely studied model substrate for functionalized olefins.

Scheme 1. Schematic View of the Investigated Catalytic reaction; I: Styrene; II Branched Aldehyde, and III: Linear Aldehyde

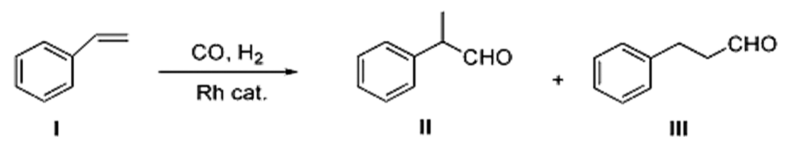

The catalytic tests were performed using a stainless-steel autoclave equipped with a glass device, and the reaction products were analyzed by GC (see the Supporting Information for details). Briefly, the sample is introduced into the glass vessel containing styrene in anhydrous toluene. The vessel is then transferred into a stainless-steel reactor and pressurized with syngas at $4-8 \mathrm{MPa}$ and heated at $50-120{ }^{\circ} \mathrm{C}$ (see Tables S2 and S3).

In the metalated MnMOFs, the atomically dispersed active sites are anchored to the organic linkers, and therefore will be affected by the chemical and structural properties of micropores. To investigate how this unique environment affects the chemical activity of the catalytic sites, we also synthesized and examined the homogeneous counterparts of $\mathrm{M} / \mathrm{MnMOF}$ moieties (vide infra).

First, we tested the catalytic activity of the metalated M/ MnMOF samples. In the case of $\mathrm{Rh} / \mathrm{MnMOF}$, a first oxoexperiment was carried out at $8 \mathrm{MPa}$ of syngas $\left(\mathrm{CO} / \mathrm{H}_{2}=1\right)$ and $50{ }^{\circ} \mathrm{C}$ for $18 \mathrm{~h}$ with a substrate to rhodium catalyst molar ratio of 1000: the substrate conversion was practically negligible (6\%), with, almost, exclusive formation of branched aldehyde II (exp. 1, Table S2). On the other hand, by increasing the reaction temperature to $80^{\circ} \mathrm{C}$, the $\mathrm{Rh} / \mathrm{MnMOF}$ catalyst becomes very active, showing complete substrate conversion and total chemoselectivity. The regioselectivity toward branched aldehyde II, even if lower than that exhibited by homogeneous modified rhodium carbonyl complexes, ${ }^{2,30,31}$ was (69\%) (Tables 1, S2 and S4) comparable to other heterogeneous rhodium catalysts. ${ }^{17,18,32}$ Therefore, we conclude that the pore structure of Rh/MnMOF does not have a significant effect on the regioselectivity of the reaction. We note that the recovered solid catalyst was reused in two consecutive experiments and the catalytic activity (both conversion and selectivity) remained unchanged. To further investigate the recyclability and the presence of deactivation processes, the $\mathrm{Rh} / \mathrm{MnMOF}$ was tested for shorter reaction times (e.g., conversion $<100 \%{ }^{33}$ ). After $3 \mathrm{~h}$, the substrate conversion was $78.5 \%$, and the regioselectivity toward branched aldehyde II was $56.9 \%$. The catalyst was then recovered and reused in two consecutive experiments $(3 \mathrm{~h}$ each): the substrate conversion was slightly reduced after the third cycle, but the regioselectivity was maintained (Table S2). An attempt to reduce the syngas pressure to $4 \mathrm{MPa}$ was not successful: after $18 \mathrm{~h}$ at $80{ }^{\circ} \mathrm{C}$, the substrate conversion was only $60 \%$, and in two consecutive recycle experiments, the catalytic activity dramatically decreased affording $18 \%$ and $11 \%$ conversion, respectively (exp. 5-7, Table S2); moreover, the regioselectivity toward branched aldehyde II, the main reaction 
Table 1. Summary of the Catalytic Results of Hydroformylation of Styrene of Rh/MnMOF, Co/MnMOF, $\mathrm{Rh} / \mathrm{E}-\mathrm{LH}_{2}$, and Co/ E- $\mathrm{LH}_{2}$ (the Substrate/Metal Molar Ratio is 1000 and 50, Respectively, for Rh and Co-Containing Catalysts)

\begin{tabular}{|c|c|c|c|c|c|c|}
\hline sample & exp. & $T\left({ }^{\circ} \mathrm{C}\right)$ & time $(\mathrm{h})$ & conversion (\%) & $b$-aldehyde (II, \%) & $n$-aldehyde (III, \%) \\
\hline \multirow[t]{4}{*}{$\mathrm{Rh} / \mathrm{MnMOF}$} & 1 & 80 & 18 & 100 & 69 & 31 \\
\hline & $2^{a}$ & 80 & 18 & 100 & 69 & 31 \\
\hline & $3^{a}$ & 80 & 18 & 100 & 69 & 31 \\
\hline & 4 & 80 & 3 & 78.5 & 56.9 & 21.8 \\
\hline $\mathrm{Co} / \mathrm{MnMOF}$ & 5 & 120 & 24 & 60 & 24 & 6 \\
\hline \multirow[t]{2}{*}{$\mathrm{Rh} / \mathrm{E}-\mathrm{LH}_{2}$} & 6 & 80 & 18 & 100 & 70 & 30 \\
\hline & $7^{a}$ & 80 & 18 & 60 & 49 & 11 \\
\hline \multirow[t]{2}{*}{$\mathrm{Co} / \mathrm{E}_{-} \mathrm{LH}_{2}$} & 8 & 120 & 24 & 100 & 54 & 46 \\
\hline & $9^{a}$ & 120 & 24 & 77 & 39 & 38 \\
\hline
\end{tabular}

${ }^{a}$ Reaction carried out using the catalyst recovered from the previous cycle.

product, was lower than that observed at $8 \mathrm{MPa}$. On the basis of the generally accepted mechanism of rhodium-catalyzed hydroformylation, a plausible explanation for this result could be that at $4 \mathrm{MPa}$ of syngas, the migratory insertion on a $\mathrm{CO}$ bound to the rhodium site to form the branched acyl rhodium species is slower than that at $8 \mathrm{MPa}$ and, as a consequence, it undergoes a $\beta$-hydride elimination giving the linear $\mathrm{Rh}$-alkyl species that subsequently affords the corresponding linear aldehyde. $^{34}$ Very recently, deuteroformylation studies on styrene at 40 and 80 psia $\mathrm{CO}$, in the presence of the homogeneous catalyst rhodium bis(diazaphospholane), demonstrated that deuterium scrambling, and hence Rh-alkyl isomerization, is inhibited by increased $\mathrm{CO}$ pressure and decreased temperature. ${ }^{34}$ In particular, the reactions at low $\mathrm{CO}$ pressure $(20$ psia, $313 \mathrm{~K})$ allowed for greater isomerization of the catalyst to the thermodynamically favored linear acyl intermediate, while at higher CO pressures (115-200 psia), the isomerization was slowed, thus favoring the branched pathway. ${ }^{34}$ Moreover, isomerization by the reversion of the acyl complex to the alkyl one is slower at high pressures because such reversion requires $\mathrm{CO}$ dissociation. ${ }^{34}$ Despite the fact that the organic phase remained colorless, after each experiment, we evaluated the possibility of metal leaching from $\mathrm{Rh} / \mathrm{MnMOFs}$. The organic phase recovered after the first reaction the $\mathrm{Rh} / \mathrm{MnMOF}$ was used as a catalyst for the homogeneous hydrogenation of undec-1-ene in toluene: after $18 \mathrm{~h}$ at $80^{\circ} \mathrm{C}$ and $8 \mathrm{MPa}$ of hydrogen, undecane was formed in negligible amounts (4\%), thus excluding rhodium leaching. In each of the experiments, neither ethylbenzene nor alcohols were detected in the reaction mixtures, and both the chemoand regio-selectivity remained practically unchanged. Furthermore, the catalytic performance of $\mathrm{Rh} / \mathrm{MnMOF}$ is comparable to those of the best-performing literature examples of MOFsupported $\mathrm{Rh}$ catalysts for olefin hydroformylation (i.e., total chemoselectivity, $70 \%$ yield of branched aldehyde). ${ }^{16-18}$ However, in the case of $\mathrm{Rh} / \mathrm{MnMOF}$, the active sites are precisely located and atomically dispersed in the porous framework. This feature limits the possibility of chemical/steric environment inhomogeneity related to agglomeration/size distribution of NPs within porous structures. . $20,35,36^{\text {We }}$ also assessed the catalytic performance of $\mathrm{Co} / \mathrm{MnMOF}$ toward the hydroformylation of styrene. The first oxo-experiment was carried out at $8 \mathrm{MPa}$ of syngas $\left(\mathrm{CO} / \mathrm{H}_{2}=1\right)$ and $80{ }^{\circ} \mathrm{C}$ for 18 $\mathrm{h}$ and at $120{ }^{\circ} \mathrm{C}$ for $24 \mathrm{~h}$ with a substrate to cobalt catalyst molar ratio of $1000: 1$, but the substrate conversion was negligible. Therefore, we lowered the substrate to cobalt catalyst molar ratio to 50:1. After $24 \mathrm{~h}$, we obtained $60 \%$ conversion with only partial chemoselectivity $(30 \%$ of ethylbenzene was detected). It is well known that Co complexes have a lower regioselectivity compared to that of $\mathrm{Rh}$ complexes. In fact, under our experimental conditions, we obtained a branched to linear aldehyde molar ratio of 24:6 (Table S2). Moreover, the recovered solid catalyst was recycled in a consecutive experiment, but the catalytic activity was negligible.

Finally, to investigate how the microporous environment affects the chemical activity of the catalytic sites, we synthesized and tested the catalytic activity of the homogeneous counterparts of $\mathrm{M} / \mathrm{MnMOF}$ moieties $\left(\mathrm{M} / \mathrm{E}-\mathrm{LH}_{2}, \mathrm{M}=\right.$ $\mathrm{Rh}$ or $\mathrm{Co})$. The $\mathrm{M} / \mathrm{E}-\mathrm{LH}_{2}$ coordination compound was obtained via the esterification of the ligand molecule (to obtain E- $\mathrm{LH}_{2}$ ), and the subsequent exposure to $\mathrm{Rh}$ and Co precursors (synthetic procedure and metal quantification are reported in the Experimental section). The $\mathrm{M} / \mathrm{E}-\mathrm{LH}_{2}$-metalated ester derivatives of the ligand molecule were tested employing the same experimental conditions of their heterogeneous counterpart (Table S3). In the case of $\mathrm{Rh} / \mathrm{E}-\mathrm{LH}_{2}$ sample, the first oxoexperiment was carried out at $8 \mathrm{MPa}$ of syngas $\left(\mathrm{CO} / \mathrm{H}_{2}=1\right)$ and $80{ }^{\circ} \mathrm{C}$ for $18 \mathrm{~h}$ with a substrate to rhodium catalyst molar ratio of 1000:1. The catalyst showed a catalytic activity comparable to that of the $\mathrm{Rh} / \mathrm{MnMOF}$ sample (total conversion, $70 \%$ of regioselectivity toward the branched aldehyde). However, the resulting organic was pale-yellow colored, suggesting probable leaching of $\mathrm{Rh}$ species. In order to confirm this hypothesis, the catalyst was recovered and the organic solution was tested for the hydrogenation of styrene (5 $\mathrm{MPa}$ of $\mathrm{H}_{2}, 80^{\circ} \mathrm{C}$ ). After $4 \mathrm{~h}$, the styrene conversion was $73 \%$ with the formation of ethylbenzene, confirming the presence of active $\mathrm{Rh}$ species within the organic solution. Therefore, in order to reduce the leaching of $\mathrm{Rh}$ species, we lowered the reaction temperature to $60{ }^{\circ} \mathrm{C}$. Surprisingly, the styrene conversion was quantitative and the regioselectivity toward the branched aldehyde was exceptionally high (9:1). However, even in this case, we observed the leaching of Rh species, and the recycled catalyst showed low conversion (49\%) and very low regioselectivity toward the branched aldehyde (11:38). In the case of $\mathrm{Co} / \mathrm{E}^{-} \mathrm{LH}_{2}$ sample, the first oxo-experiment was carried out at $8 \mathrm{MPa}$ of syngas $\left(\mathrm{CO} / \mathrm{H}_{2}=1\right)$ and $120{ }^{\circ} \mathrm{C}$ for $18 \mathrm{~h}$ with a substrate to cobalt catalyst molar ratio of $50: 1$. We observed complete substrate conversion and total chemoselectivity but with low regioselectivity toward branched aldehyde II (54\%). Similar to the $\mathrm{Rh} / \mathrm{E}-\mathrm{LH}_{2}$ sample, we observed leaching of the Co species, and the recycled catalyst showed lower substrate conversion (77\%) and regioselectivity (almost equimolar formation of branched and linear aldehydes). The instability of $\mathrm{M} / \mathrm{E}-\mathrm{LH}_{2}$ samples highlights 
the role of the MOF structure in anchoring the active sites and in preventing the metal leaching, as previously reported for molecular catalysts heterogenized on MOFs. ${ }^{37-39}$ Moreover, the crystalline powder nature of $\mathrm{M} / \mathrm{MnMOF}$ facilitates the recovery and reusability of the catalyst. To ascertain the reduction of the catalytic performance of $\mathrm{Co} / \mathrm{MnMOF}$ and the stability of the catalytic activity of $\mathrm{Rh} / \mathrm{MnMOF}$, we examined the structure and composition of M/MnMOFs. EDX and ICP analyses (Table S1) confirmed that the weight ratio between $\mathrm{Mn}$ and $\mathrm{Rh}$ is maintained $(\mathrm{Mn} / \mathrm{Rh}=1.3)$. Conversely, in the case of $\mathrm{Co} / \mathrm{MnMOF}$, ICP confirmed the leakage of both Mn and Co (the weight percentage changes, respectively, from 9.65 and 3.45 to 5.5 and $2.2 \%$ after the first catalytic cycle and to 1.4 and $0.83 \%$ after the recyclability test). We also investigated the morphology of crystals before and after catalysis. The morphology of pristine MnMOF crystals consists of rhombic plates of ca. 150-300 $\mu \mathrm{m}$ (Figure S8a). Indeed, SEM experiments showed that the crystal shape is retained after metalation (Figures S8b and S9a). For the exhausted materials, only in the case of $\mathrm{Rh} / \mathrm{MnMOF}$, the crystal morphology was unchanged (Figure S8c); the used $\mathrm{Co} /$ MnMOF is composed of undefined particles (Figure S9b). The structural integrity of $\mathrm{M} / \mathrm{MnMOF}$ after catalytic tests was also investigated by Raman spectroscopy (Figures 4 and S4) and XRD (Figures 2 and S1). Combined Raman spectra and XRD patterns of used $\mathrm{Rh} / \mathrm{MnOF}$ suggest that the structural integrity is maintained. Moreover, X-ray photoemission spectroscopy (XPS, Figure S5) showed that the Rh $3 \mathrm{~d}$ photoemission peak of the used material is centered at $308 \mathrm{eV}$, as in the fresh $\mathrm{Rh} /$ MnMOF. Conversely, the Raman spectrum of used $\mathrm{Co} /$ MnMOF suggests the degradation of the MOF structure (Figure S4, broad bands in the $1200-1600 \mathrm{~cm}^{-1}$ spectral region), and the $\mathrm{XRD}$ pattern shows a severe loss of crystallinity and the formation of $\mathrm{Co}_{3} \mathrm{O}_{4}$ (Figure S1). Finally, we assessed the porosity of the exhausted $\mathrm{Rh} / \mathrm{MnMOF}$ catalyst by gas adsorption isotherm analysis. The $77 \mathrm{~K} \mathrm{~N}_{2}$ adsorption isotherm of the used $\mathrm{Rh} / \mathrm{MnMOF}$ catalyst showed type I isotherm (Figure 3), indicating that the microporosity is maintained, despite the reduction of the calculated BET surface area $\left(444 \pm 1 \mathrm{~m}^{2} \mathrm{~g}^{-1}\right)$ that could be associated with the presence of residual reagent/products in the pores. Moreover, the calculated pore size distribution is essentially unchanged compared to that of the fresh sample (Figure S7).

\section{CONCLUSIONS}

In conclusion, exploiting a postsynthetic metalation strategy, we have introduced in the structure of MnMOF two atomically dispersed catalytic sites based on $\mathrm{Rh}(\mathrm{I})$ and $\mathrm{Co}(\mathrm{II})$. These metal centers were tested as hydroformylation catalysts, a reaction typically performed by homogeneous catalysts or Rhsupported nanoparticles. For comparison, we investigated the homogeneous counterpart of the active species in the metalated MOF. By anchoring the catalytic sites in the crystal structure of the MOF, it was possible to combine the performances of a homogeneous catalysts with the advantages of a heterogeneous catalyst (i.e., recyclability, separation from the reaction mixture). The best-performing investigated material was the $\mathrm{Rh} / \mathrm{MnMOF}$. In this case, working at 80 ${ }^{\circ} \mathrm{C}$ and $8 \mathrm{MPa}$ of syngas, we obtained the complete substrate (styrene) conversion, total chemoselectivity, high regioselectivity toward the branched aldehyde II (70\%), and good recyclability over three catalytic cycles. MOF-based materials were previously tested as hydroformylation catalysts only in combination with metallic nanoparticles. ${ }^{16-18}$ The possibility of having a high loading (i.e., 5.4 wt \% of Rh) of active species precisely located within the porous framework, without the problem of blocking the pore access in the presence of nanoparticles, could be exploited in different catalytic processes, both in liquid and, especially, in the gas phase. Furthermore, comparing our data with the performance of the homogeneous catalyst suggests that the mass transport through the MOF channels is not a significant limiting factor for the reaction rate. This work provides insights into the heterogenization of an important homogeneous catalytic process within a MOF structure and paves the way for the further development of atomically dispersed catalysts based on postsynthetic metalated MOFs.

\section{EXPERIMENTAL SECTION}

4.1. Materials and Methods. 4.1.1. Materials Synthesis. Unless otherwise stated, all chemicals were obtained from commercial sources (Sigma-Aldrich, Alfa Aesar) and used as received.

4.1.2. Synthesis of $\operatorname{MnMOF}\left(\left[M n_{3}(L)_{2}\left(L^{\prime}\right)\right]\right)$. The ligand $\mathrm{LH}_{2}$ was synthesized following a previously reported procedure. ${ }^{22} \mathrm{MnMOF}$ was synthesized as previously reported by Bloch et al. ${ }^{23}$ Briefly, $24.7 \mathrm{mg}$ of $\mathrm{MnCl}_{2} \cdot 4 \mathrm{H}_{2} \mathrm{O}$ and $31.6 \mathrm{mg}$ of $\mathrm{LH}_{2}(1.7: 1$ molar ratio) were dissolved in $6 \mathrm{~mL}$ of dimethylformamide $(\mathrm{DMF}) / \mathrm{H}_{2} \mathrm{O}(2: 1 \mathrm{vol}$ ratio $)$ and heated in oven at $100{ }^{\circ} \mathrm{C}$ for $48 \mathrm{~h}$ in a screw-cap vial. The obtained colorless crystals (yield: 70\%) were washed in DMF $(\times 3)$ and methanol (X5) and then dried under vacuum for $12 \mathrm{~h}$. The schematic view of the MOF structure is reported in Figure 1.

4.1.3. Postsynthetic Metalation of MnMOF. Rh/MnMOF: the assynthesized material, MnMOF (25 mg), was solvent-exchanged with dry acetonitrile. The solvent was replenished five times and the crystals were allowed to soak in dry acetonitrile for $10 \mathrm{~min}$ in-between each wash. Then, $18 \mathrm{mg}$ of $\left[\mathrm{Rh}(\mathrm{CO})_{2} \mathrm{Cl}\right]_{2}$ was added to the acetonitrile dispersion of MnMOF, and the resulting mixture was left at RT for $60 \mathrm{~h}$. Then, the solvent was exchanged for fresh dry acetonitrile $(\times 7)$. The solvent was decanted, and the pale-yellow wet crystals of $\mathrm{Rh} / \mathrm{MnMOF}$ were dried under vacuum for $12 \mathrm{~h}$. The presence of $\mathrm{Rh}(\mathrm{I})$ in the final material was confirmed by XPS (Figure S5).

Co/MnMOF: the as-synthesized material, MnMOF (25 mg), was solvent-exchanged with methanol. The solvent was replenished five times and the crystals were allowed to soak in methanol for $10 \mathrm{~min}$ inbetween each wash. Then, $23 \mathrm{mg}$ of $\mathrm{CoCl}_{2} \cdot\left(\mathrm{H}_{2} \mathrm{O}\right)_{6}$ was added to the methanol dispersion of $\mathrm{MnMOF}$, and the resulting mixture was left at RT for $48 \mathrm{~h}$. Then, the solvent was exchanged for fresh methanol $(\times 7)$. The solvent was decanted, and the blue wet crystals of $\mathrm{Co} /$ MnMOF were dried under vacuum for $12 \mathrm{~h}$.

4.1.4. Esterification of $\mathrm{LH}_{2} \cdot 0.329 \mathrm{mg}(0.75 \mathrm{mmol})$ of $\mathrm{LH}_{2}$ was suspended in $5 \mathrm{~mL}$ of degassed ethanol under an argon atmosphere. To this mixture, $0.544 \mathrm{~g}(8 \mathrm{mmol})$ of sodium ethoxide was added, and the reaction was maintained with magnetic stirring under reflux conditions until the disappearance of the initial reagent, as determined by thin-layer chromatography analysis, typically for $24 \mathrm{~h}$. After cooling down, the reaction mixture was neutralized with $1 \mathrm{M} \mathrm{HCl}$, and a white precipitate appeared, which was filtered, washed once with 10 $\mathrm{mL}$ of $2 \mathrm{M} \mathrm{NaOH}$, and then thoroughly with water. Drying under vacuum yielded the esterified product (hereafter called $\mathrm{E}-\mathrm{LH}_{2}$ ) in quantitative yields. ${ }^{1} \mathrm{H}$ NMR (DMSO- $\left.d_{6}, 298 \mathrm{~K}, \delta \mathrm{ppm}\right): 7.98$ (d, $J=$ $8.3 \mathrm{~Hz}, 4 \mathrm{H}), 7.41(\mathrm{~d}, J=8.3 \mathrm{~Hz}, 4 \mathrm{H}), 6.28(\mathrm{~s}, 2 \mathrm{H}), 3.42(\mathrm{c}, J=7.1$ $\mathrm{Hz}, 4 \mathrm{H}) 2.15(\mathrm{~s}, 6 \mathrm{H}), 2.08(\mathrm{~s}, 6 \mathrm{H}), 1.05(\mathrm{t}, J=7.0 \mathrm{~Hz}, 6 \mathrm{H})$. Reaction scheme and ${ }^{1} \mathrm{H}$ NMR spectrum of esterified $\mathrm{LH}_{2}$ are presented in Figure S9.

4.1.5. Postsynthetic Metalation of Esterified $\mathrm{LH}_{2} \cdot \mathrm{Rh} / \mathrm{E}-\mathrm{LH}_{2}$ : the as-synthesized material, E- $\mathrm{LH}_{2}(50 \mathrm{mg})$, was washed five times with dry acetonitrile. Then, $36 \mathrm{mg}$ of $\left[\mathrm{Rh}(\mathrm{CO})_{2} \mathrm{Cl}\right]_{2}$ was added to the acetonitrile dispersion of $\mathrm{E}-\mathrm{LH}_{2}$, and the resulting mixture was left at RT for $60 \mathrm{~h}$. Then, the powder was washed with fresh dry acetonitrile $(\times 7)$. The solvent was decanted and the pale-yellow powder of Rh/E- 
$\mathrm{LH}_{2}$ was dried under vacuum for $12 \mathrm{~h}$. The amount of Co in the final product was quantified by ICP analysis ( $\mathrm{Rh} / \mathrm{E}-\mathrm{LH}_{2}: 13.23 \mathrm{Rh}$ wt \%).

$\mathrm{Co} / \mathrm{E}-\mathrm{LH}_{2}$ : the as-synthesized material, $\mathrm{E}-\mathrm{LH}_{2}(50 \mathrm{mg})$, was washed five times with methanol. Then, $23 \mathrm{mg}$ of $\mathrm{CoCl}_{2} \cdot\left(\mathrm{H}_{2} \mathrm{O}\right)_{6}$ was added to the methanol dispersion of $\mathrm{E}-\mathrm{LH}_{2}$, and the resulting mixture was left at RT for $48 \mathrm{~h}$. Then, the powder was washed with fresh methanol $(\times 7)$. The solvent was decanted, and the blue powder of $\mathrm{Co} / \mathrm{E}-\mathrm{LH}_{2}$ was dried under vacuum for $12 \mathrm{~h}$. The amount of Co in the final product was quantified by ICP analysis $\left(\mathrm{Co} / \mathrm{E}-\mathrm{LH}_{2}: 4.69 \mathrm{Co}\right.$ wt \%).

4.2. Characterization Techniques. Powder XRD patterns were collected on a Bruker D8 Advance diffractometer (capillary) equipped with a $\mathrm{Cu} \mathrm{K} \alpha$ source $(\lambda=1.5418 \AA)$.

Scanning electron microscopy and EDX spectra were acquired using a field-emission electron source equipped with a GEMINI column (Zeiss Supra VP35).

Characterization by Raman spectroscopy was performed using a ThermoFisher DXR Raman microscope. The spectra were recorded using a laser with an excitation wavelength of $532 \mathrm{~nm}(1 \mathrm{~mW})$, focused on the sample with a 10X LWD objective (Olympus).

Gas adsorption isotherm measurements were performed on an ASAP 2020 Surface Area and Pore Size Analyzer. Samples were activated by heating in vacuum at $120{ }^{\circ} \mathrm{C}$ for $12 \mathrm{~h}$. UHP-grade (99.999\%) $\mathrm{N}_{2}$ and $\mathrm{He}$ were used for all measurements. The temperatures were maintained at $77 \mathrm{~K}$ (liquid nitrogen bath).

XPS spectra were acquired on a custom-designed ultrahigh vacuum system equipped with an Omicron EA125 electron analyzer, a nonmonochromatized $\mathrm{Al} \mathrm{K} \alpha$ source (Omicron DAR 400, $1486.6 \mathrm{eV}$ ), and a flood gun for charge neutralization (Henniker Scientific, FS40A1), as MOFs are moderately insulating. The MOF samples were dispersed in methanol and drop-casted on polished $\mathrm{Cu}$ disks, obtaining homogeneous thin films. The measurements of the asprepared materials were taken after short annealing at $150{ }^{\circ} \mathrm{C}$ in ultrahigh vacuum to remove most adsorbed species (water and contamination debris). The calibration of the $\mathrm{BE}$ scale was done using the $\mathrm{C} 1 \mathrm{~s}$ peak as the reference. The photoemission lines were separated into individual components (after Shirley background removal) using symmetrical Voigt functions and nonlinear least squares routines for $\chi^{2}$ minimization.

4.3. Hydroformylation of Styrene. The reactions were carried out using a stainless-steel autoclave equipped with a glass device that is described in detail in the Supporting Information. In a typical run, the basket containing the catalytic complex was introduced, under a nitrogen purge, in a glass vessel containing styrene in anhydrous toluene. The vessel was transferred into a $150 \mathrm{~mL}$ stainless-steel reactor, which was pressurized with syngas at $4-8 \mathrm{MPa}$ and heated at 50-120 ${ }^{\circ} \mathrm{C}$ for $18-24 \mathrm{~h}$ (see Tables S2 and S3). After cooling at room temperature, the residual gases were released, and the reaction products were analyzed by GC. The basket containing the catalyst was removed from the glass vial and the catalyst was washed several times with anhydrous toluene, dried under vacuum, and reused in recycle experiments.

\section{ASSOCIATED CONTENT}

\section{(s) Supporting Information}

The Supporting Information is available free of charge at https://pubs.acs.org/doi/10.1021/acsami.0c17073.

Catalytic test description and results; EDX and ICP-MS results; MnMOF and Co/MnMOF XRD patterns, $77 \mathrm{~K}$ $\mathrm{N}_{2}$ adsorption isotherm and Raman spectra; derivation of BET surface area; XPS data; pore size distribution; SEM micrographs; and ${ }^{1} \mathrm{H}$ NMR spectrum of the esterified ligand (PDF)

\section{AUTHOR INFORMATION}

\section{Corresponding Authors}

Francesco Carraro - Department of Chemical Sciences and INSTM Unit, University of Padova, 35131 Padova, Italy; Institute of Physical and Theoretical Chemistry, Graz University of Technology, Graz 8010, Austria; (1) orcid.org/ 0000-0001-8485-4676; Email: Francesco.carraro@ tugraz.at

Stefano Agnoli - Department of Chemical Sciences and INSTM Unit, University of Padova, 35131 Padova, Italy; ○ orcid.org/0000-0001-5204-5460;

Email: stefano.agnoli@unipd.it

\section{Authors}

Panjuan Tang - Department of Chemical Sciences and INSTM Unit, University of Padova, 35131 Padova, Italy

Stefano Paganelli - Dipartimento di Scienze Molecolari e Nanosistemi, Università Ca' Foscari Venezia, 30172 Venezia Mestre, Italy

Matias Blanco - Department of Chemical Sciences and INSTM Unit, University of Padova, 35131 Padova, Italy

Raffaele Riccò - Institute of Physical and Theoretical Chemistry, Graz University of Technology, Graz 8010, Austria; 1 orcid.org/0000-0002-0296-9398

Carla Marega - Department of Chemical Sciences and INSTM Unit, University of Padova, 35131 Padova, Italy

Denis Badocco - Department of Chemical Sciences and INSTM Unit, University of Padova, 35131 Padova, Italy

Paolo Pastore - Department of Chemical Sciences and INSTM Unit, University of Padova, 35131 Padova, Italy

Christian J. Doonan - Department of Chemistry, University of Adelaide, Adelaide, South Australia 5005, Australia; (1) orcid.org/0000-0003-2822-0956

Complete contact information is available at: https://pubs.acs.org/10.1021/acsami.0c17073

\section{Author Contributions}

The manuscript was written through contributions of all authors. All authors have given approval to the final version of the manuscript.

\section{Notes}

The authors declare no competing financial interest.

\section{ACKNOWLEDGMENTS}

The preliminary results that led to this work were reported in the PhD Thesis by F.C.: Multiscale design of nanostructured materials for heterogeneous catalysis. ${ }^{40}$ F.C. acknowledges TU Graz for the Lead Project (LP-03). R.R. acknowledges the European Union's Horizon 2020 Research and Innovation Programme under the Marie Skłodowska-Curie Grant 748649. F.C. and S.A. acknowledge the grant Attrezzature scientifiche finalizzate alla ricerca-Bando 2012. S.A. acknowledges the U.S.-Italy Fulbright Commission for financial support. P.T. acknowledges financial support by the Chinese Scholar Council.

\section{ABBREVIATIONS}

MOF, metal-organic framework

$\mathrm{MnMOF},\left[\mathrm{Mn}_{3}(\mathrm{~L})_{2}\left(\mathrm{~L}^{\prime}\right)\right]$

$\mathrm{LH}_{2}$, bis(4-(4-carboxyphenyl)-1H-3,5-dimethylpyrazolyl)methane 
$\mathrm{M} / \mathrm{MnMOF}$ with $\mathrm{M}=\mathrm{Rh}$ or $\mathrm{Co}$, postsynthetic metalated $\mathrm{MnMOF}$

E- $\mathrm{LH}_{2}$, esterified $\mathrm{LH}_{2}$

$\mathrm{M} / \mathrm{E}-\mathrm{LH}_{2}$ with $\mathrm{M}=\mathrm{Rh}$ or $\mathrm{Co}$, postsynthetic metalated $\mathrm{E}$ $\mathrm{LH}_{2}$

SEM, scanning electron microscopy

XPS, X-ray photoemission spectroscopy

EDX, energy-dispersive X-ray spectroscopy

$\mathrm{XRD}, \mathrm{X}$-ray diffraction

ICP-MS, inductively coupled plasma-mass spectrometry

GC, gas chromatography

\section{REFERENCES}

(1) Cornils, B.; Herrmann, W. A. Applied Homogeneous Catalysis with Organometallic Compounds, 2nd ed. Wiley-VCH: Weinheim, 2002.

(2) van Leeuwen, P. W. N. M.; Claver, C. Rhodium Catalyzed Hydroformylation; Springer, 2002.

(3) Franke, R.; Selent, D.; Börner, A. Applied Hydroformylation. Chem. Rev. 2012, 112, 5675.

(4) Corma, A.; García, H.; Llabrés i Xamena, F. X. Engineering Metal Organic Frameworks for Heterogeneous Catalysis. Chem. Rev. 2010, 110, 4606-4655.

(5) Zhao, X. S.; Bao, X. Y.; Guo, W.; Lee, F. Y. Immobilizing Catalysts on Porous Materials. Mater. Today 2006, 9, 32-39.

(6) Barbaro, P., Liguori, F. Heterogenized Homogeneous Catalysts for Fine Chemicals Production: Materials and Processes; Springer Netherlands: Dordrecht, 2010.

(7) Li, C.; Wang, W.; Yan, L.; Ding, Y. A Mini Review on Strategies for Heterogenization of Rhodium-Based Hydroformylation Catalysts. Front. Chem. Sci. Eng. 2018, 12, 113-123.

(8) Khokhar, M. D.; Shukla, R. V.; Jasra, R. V. Rh Complex Encapsulated Hexagonal Mesoporous Silica as an Efficient Heterogeneous Catalyst for the Selective Hydroformylation of Styrene. React. Kinet., Mech. Catal. 2014, 114, 265.

(9) Rodríguez-reinoso, F. The Role of Carbon Materials in Heterogeneous Catalysis. Carbon 1998, 36, 159-175.

(10) Kosinov, N.; Liu, C.; Hensen, E. J. M.; Pidko, E. A. Engineering of Transition Metal Catalysts Confined in Zeolites. Chem. Mater. 2018, 30, 3177-3198.

(11) Lee, J.; Farha, O. K.; Roberts, J.; Scheidt, K. A.; Nguyen, S. T.; Hupp, J. T. Metal-Organic Framework Materials as Catalysts. Chem. Soc. Rev. 2009, 38, 1450-1459.

(12) Doonan, C. J.; Sumby, C. J. Metal-Organic Framework Catalysis. CrystEngComm 2017, 19, 4044-4048.

(13) Genna, D. T.; Wong-Foy, A. G.; Matzger, A. J.; Sanford, M. S. Heterogenization of Homogeneous Catalysts in Metal-Organic Frameworks via Cation Exchange. J. Am. Chem. Soc. 2013, 135, 10586-10589.

(14) Qin, J.-S.; Yuan, S.; Lollar, C.; Pang, J.; Alsalme, A.; Zhou, H.C. Stable Metal-Organic Frameworks as a Host Platform for Catalysis and Biomimetics. Chem. Commun. 2018, 54, 4231-4249.

(15) Dhakshinamoorthy, A.; Asiri, A. M.; Garcia, H. Tuneable Nature of Metal Organic Frameworks as Heterogeneous Solid Catalysts for Alcohol Oxidation. Chem. Commun. 2017, 53, 1085110869.

(16) Van Vu, T.; Kosslick, H.; Schulz, A.; Harloff, J.; Paetzold, E.; Schneider, M.; Radnik, J.; Steinfeldt, N.; Fulda, G.; Kragl, U. Selective Hydroformylation of Olefins over the Rhodium Supported Large Porous Metal-Organic Framework MIL-101. Appl. Catal., A 2013, 468, 410-417.

(17) Van Vu, T.; Kosslick, H.; Schulz, A.; Harloff, J.; Paetzold, E.; Radnik, J.; Kragl, U.; Fulda, G.; Janiak, C.; Tuyen, N. D. Hydroformylation of Olefins over Rhodium Supported Metal-Organic Framework Catalysts of Different Structure. Microporous Mesoporous Mater. 2013, 177, 135-142.

(18) Hou, C.; Zhao, G.; Ji, Y.; Niu, Z.; Wang, D.; Li, Y. Hydroformylation of Alkenes over Rhodium Supported on the Metal-Organic Framework ZIF-8. Nano Res. 2014, 7, 1364-1369.
(19) Dhakshinamoorthy, A.; Garcia, H. Catalysis by Metal Nanoparticles Embedded on Metal-Organic Frameworks. Chem. Soc. Rev. 2012, 41, 5262-5284.

(20) Lu, G.; Li, S.; Guo, Z.; Farha, O. K.; Hauser, B. G.; Qi, X.; Wang, Y.; Wang, X.; Han, S.; Liu, X.; DuChene, J. S.; Zhang, H.; Zhang, Q.; Chen, X.; Ma, J.; Loo, S. C. J.; Wei, W. D.; Yang, Y.; Hupp, J. T.; Huo, F. Imparting Functionality to a Metal-Organic Framework Material by Controlled Nanoparticle Encapsulation. Nat. Chem. 2012, 4, 310-316.

(21) Fei, H.; Cohen, S. M. A Robust, Catalytic Metal-Organic Framework with Open 2,2'-Bipyridine Sites. Chem. Commun. 2014, 50, 4810-4812.

(22) Bloch, W. M.; Doonan, C. J.; Sumby, C. J. Using Hinged Ligands to Target Structurally Flexible Copper(II) MOFs. CrystEngComm 2013, 15, 9663-9671.

(23) Bloch, W. M.; Burgun, A.; Coghlan, C. J.; Lee, R.; Coote, M. L.; Doonan, C. J.; Sumby, C. J. Capturing Snapshots of Post-Synthetic Metallation Chemistry in Metal-Organic Frameworks. Nat. Chem. 2014, 6, 906-912.

(24) Burgun, A.; Coghlan, C. J.; Huang, D. M.; Chen, W.; Horike, S.; Kitagawa, S.; Alvino, J. F.; Metha, G. F.; Sumby, C. J.; Doonan, C. J. Mapping-Out Catalytic Processes in a Metal-Organic Framework with Single-Crystal X-Ray Crystallography. Angew. Chem. 2017, 129, $8532-8536$.

(25) Tang, C.-W.; Wang, C.-B.; Chien, S.-H. Characterization of Cobalt Oxides Studied by FT-IR, Raman, TPR and TG-MS. Thermochim. Acta 2008, 473, 68-73.

(26) Buciuman, F.; Patcas, F.; Craciun, R.; Zahn, D. R. T. Vibrational Spectroscopy of Bulk and Supported Manganese Oxides. Phys. Chem. Chem. Phys. 1999, 1, 185-190.

(27) Weber, W. H.; Baird, R. J.; Graham, G. W. Raman Investigation of Palladium Oxide, Rhodium Sesquioxide and Palladium Rhodium Dioxide. J. Raman Spectrosc. 1988, 19, 239-244.

(28) Feltham, R. D.; Brant, P. XPS Studies of Core Binding Energies in Transition Metal Complexes. 2. Ligand Group Shifts. J. Am. Chem. Soc. 1982, 104, 641-645.

(29) Strydom, C. A.; Strydom, H. J. X-Ray Photoelectron Spectroscopy Studies of Some Cobalt(II) Nitrate Complexes. Inorg. Chim. Acta 1989, 159, 191-195.

(30) Kamer, P. J. K.; van Leeuwen, P. W. N. M. Phosphorus(III) Ligands in Homogeneous Catalysis: Design and Synthesis; Wiley, 2012.

(31) Trzeciak, A. M.; Ziółkowski, J. J. Perspectives of Rhodium Organometallic Catalysis. Fundamental and Applied Aspects of Hydroformylation. Coord. Chem. Rev. 1999, 190-192, 883-900.

(32) Neves, Â. C. B.; Calvete, M. J. F.; Pinho e Melo, T. M. V. D.; Pereira, M. M. Immobilized Catalysts for Hydroformylation Reactions: A Versatile Tool for Aldehyde Synthesis. Eur. J. Org. Chem. 2012, 2012, 6309-6320.

(33) Schüth, F.; Ward, M. D.; Buriak, J. M. Common Pitfalls of Catalysis Manuscripts Submitted to Chemistry of Materials. Chem. Mater. 2018, 30, 3599-3600.

(34) Brezny, A. C.; Landis, C. R. Unexpected CO Dependencies, Catalyst Speciation, and Single Turnover Hydrogenolysis Studies of Hydroformylation via High Pressure NMR Spectroscopy. J. Am. Chem. Soc. 2017, 139, 2778-2785.

(35) Bell, A. T. The Impact of Nanoscience on Heterogeneous Catalysis. Science 2003, 299, 1688-1691.

(36) Zhu, Q.-L.; Xu, Q. Immobilization of Ultrafine Metal Nanoparticles to High-Surface-Area Materials and Their Catalytic Applications. Chem 2016, 1, 220-245.

(37) Yang, D.; Odoh, S. O.; Wang, T. C.; Farha, O. K.; Hupp, J. T.; Cramer, C. J.; Gagliardi, L.; Gates, B. C. Metal-Organic Framework Nodes as Nearly Ideal Supports for Molecular Catalysts: NU-1000and UiO-66-Supported Iridium Complexes. J. Am. Chem. Soc. 2015, 137, 7391-7396.

(38) Drake, T.; Ji, P.; Lin, W. Site Isolation in Metal-Organic Frameworks Enables Novel Transition Metal Catalysis. Acc. Chem. Res. 2018, 51, 2129-2138. 
(39) Viciano-Chumillas, M.; Mon, M.; Ferrando-Soria, J.; Corma, A.; Leyva-Pérez, A.; Armentano, D.; Pardo, E. Metal-Organic Frameworks as Chemical Nanoreactors: Synthesis and Stabilization of Catalytically Active Metal Species in Confined Spaces. Acc. Chem. Res. 2020, 53, 520-531.

(40) Carraro, F. Multiscale Design of Nanostructured Materials for Catalysis. Ph.D. Thesis, Doctorate School of Science and Engineering of Materials and Nanostructures, University of Padova, Italy 2018. 